\title{
Engineering glutathione biosynthesis of Saccharomyces cerevisiae increases robustness to inhibitors in pretreated lignocellulosic materials
}

\author{
Magnus Ask' ${ }^{1}$ Valeria Mapelli ${ }^{1}$, Heidi Höck ${ }^{1,2}$, Lisbeth Olsson ${ }^{1}$ and Maurizio Bettiga ${ }^{1 *}$
}

\begin{abstract}
Background: Production of bioethanol from lignocellulosic biomass requires the development of robust microorganisms that can tolerate the stressful conditions prevailing in lignocellulosic hydrolysates. Several inhibitors are known to affect the redox metabolism of cells. In this study, Saccharomyces cerevisiae was engineered for increased robustness by modulating the redox state through overexpression of GSH1, CYS3 and GLR1, three genes involved in glutathione (GSH) metabolism.
\end{abstract}

Results: Overexpression constructs were stably integrated into the genome of the host strains yielding five strains overexpressing GSH1, GSH1/CYS3, GLR1, GSH1/GLR1 and GSH1/CYS3/GLR1. Overexpression of GSH1 resulted in a 42\% increase in the total intracellular glutathione levels compared to the wild type. Overexpression of GSH1/CYS3, GSH1/ GLR1 and GSH1/CYS3/GLR1 all resulted in equal or less intracellular glutathione concentrations than overexpression of only GSH1, although higher than the wild type. GLR1 overexpression resulted in similar total glutathione levels as the wild type. Surprisingly, all recombinant strains had a lower [reduced glutathione]:[oxidized glutathione] ratio (ranging from 32-67) than the wild type strain (88), suggesting a more oxidized intracellular environment in the engineered strains. When considering the glutathione half-cell redox potential ( $\left.E_{h c}\right)$, the difference between the strains was less pronounced. Ehc for the recombinant strains ranged from -225 to $-216 \mathrm{mV}$, whereas for the wild type it was estimated to $-225 \mathrm{mV}$. To test whether the recombinant strains were more robust in industrially relevant conditions, they were evaluated in simultaneous saccharification and fermentation (SSF) of pretreated spruce. All strains carrying the GSH1 overexpression construct performed better than the wild type in terms of ethanol yield and conversion of furfural and HMF. The strain overexpressing GSH1/GLR1 produced $14.0 \mathrm{~g} \mathrm{~L}^{-1}$ ethanol in 48 hours corresponding to an ethanol yield on hexoses of $0.17 \mathrm{~g} \mathrm{~g}^{-1}$; while the wild type produced $8.2 \mathrm{~g} \mathrm{~L}^{-1}$ ethanol in 48 hours resulting in an ethanol yield on hexoses of $0.10 \mathrm{~g} \mathrm{~g}^{-1}$.

Conclusions: In this study, we showed that engineering of the redox state by modulating the levels of intracellular glutathione results in increased robustness of S. cerevisiae in SSF of pretreated spruce.

Keywords: Lignocellulose, Bioethanol, Inhibitors, Robustness, Glutathione, Redox metabolism

\section{Background}

In order to cope with the stressful conditions in industrial fermentations, robust microorganisms are needed [1]. This applies especially for the production of bioethanol from lignocellulosic feedstocks such as agricultural and forest residues. The recalcitrant nature of

\footnotetext{
* Correspondence: maurizio.bettiga@chalmers.se

${ }^{1}$ Department of Chemical and Biological Engineering, Industrial

Biotechnology, Chalmers University of Technology, Gothenburg SE-41296,

Sweden

Full list of author information is available at the end of the article
}

these materials requires harsh pretreatment methods, which aside from imposing structural changes facilitating enzymatic hydrolysis generate compounds acting as inhibitors for cellulolytic enzymes as well as microorganisms [2]. Apart from organic acids and phenolic compounds, the furan aldehydes 5-hydroxymethylfurfural (HMF) and 2-furaldehyde (furfural) stand out as particularly challenging for viable production of lignocellulosic ethanol [3]. These compounds have been shown to inhibit several enzymes in glycolysis, decrease specific ethanol production rate, affect cell growth and survival

\section{Biomed Central}


and to induce the formation of reactive oxygen species (ROS) in Saccharomyces cerevisiae, with DNA and membrane damage as a consequence [4-7]. Under anaerobic conditions, HMF and furfural are converted in situ to less toxic alcohols by $\mathrm{NAD}(\mathrm{P}) \mathrm{H}$-dependent oxidoreductases $[8,9]$. Both the detoxification of ROS and the conversion of HMF and furfural per se, create a more oxidized intracellular environment by draining the cells of reducing power in terms of $\mathrm{NAD}(\mathrm{P}) \mathrm{H}$, and potentially obliterate the antioxidant defense system of the cell [10].

Based on these evidences, we hypothesized the intracellular redox system as a potential metabolic engineering target for increasing robustness of $S$. cerevisiae to lignocellulosic inhibitors.

Glutathione (GSH) is the main antioxidant system in living cells and has been shown to be indispensable for, but not limited to, oxidative stress responses $[5,11,12]$. By virtue of its high intracellular concentration and low redox potential $(-240 \mathrm{mV}), \mathrm{GSH}$ has been regarded as a biological redox buffer maintaining redox homeostasis in spite of insults caused by oxidizing agents [13]. Its many functions include: scavenging of ROS, protection against endogenous toxic metabolites, detoxification of xenobiotics and involvement in sulfur and nitrogen metabolism (reviewed in [14]).

GSH is a tripeptide composed of cysteine, glutamate and glycine, in which the thiol $(-\mathrm{SH})$ group of the cysteine residue confers the activity to the molecule [14]. GSH occurs intracellularly in either its reduced form (GSH) or in its oxidized form (GSSG) where two GSH molecules are interlinked with a disulfide bond. Utilization of GSH results in the oxidation to its disulfide form, GSSG, from which GSH can be regenerated by the action of glutathione reductase, encoded by GLR1 [12]. Glr1p uses NADPH as reducing equivalents donor, thus maintaining a high cytosolic GSH:GSSG ratio of 30 - 100:1 [15,16].
This provides a reducing intracellular environment, which is thought to retain the oxidation-sensitive thiol groups of cysteine residues of proteins in a reduced state [17]. Overexpression of glutathione reductase from Oryza sativa and Brassica rapa in S. cerevisiae has recently been shown to increase tolerance against oxidative stress induced by $\mathrm{H}_{2} \mathrm{O}_{2}$ and abiotic stresses such as heavy metals $[18,19]$.

GSH is synthesized in two consecutive ATP-dependent reactions, outlined in Figure 1. The first step, catalyzed by $\gamma$-glutamylcysteine synthetase encoded by GSH1, has shown to be rate-limiting as overexpression of GSH2 led to unchanged levels of total glutathione, whereas overexpression of GSH1 resulted in an almost twofold increase in the intracellular GSH levels [20]. Yeast strains overexpressing GSH1 have been shown to possess higher tolerance to oxidative stress induced by $\mathrm{H}_{2} \mathrm{O}_{2}$ compared to wild type cells [21]. In addition to overexpression of GSH1, supplementation of the constituent amino acids of $\mathrm{GSH}$, and in particular cysteine, has shown to increase GSH accumulation in S. cerevisiae [22]. Increased expression of CYS3, encoding cystathionine- $\gamma$-lyase, was found in a UV-mutagenized strain of $S$. cerevisiae, which accumulated high levels of GSH [23].

In the present study, we investigated if engineering of the cell's antioxidant system by overexpression of three genes (GSH1, CYS3 and GLR1) involved in the metabolism of the endogenous antioxidant glutathione could alleviate the toxic effects induced by lignocellulosederived inhibitors, and thereby enhance robustness of $S$. cerevisiae for lignocellulosic hydrolysate fermentation. After confirming that the recombinant strains had altered redox characteristics by quantifying the intracellular glutathione levels, we show that the strains engineered for higher intracellular glutathione levels have superior robustness under process-like conditions in a simultaneous saccharification and fermentation (SSF) setup.

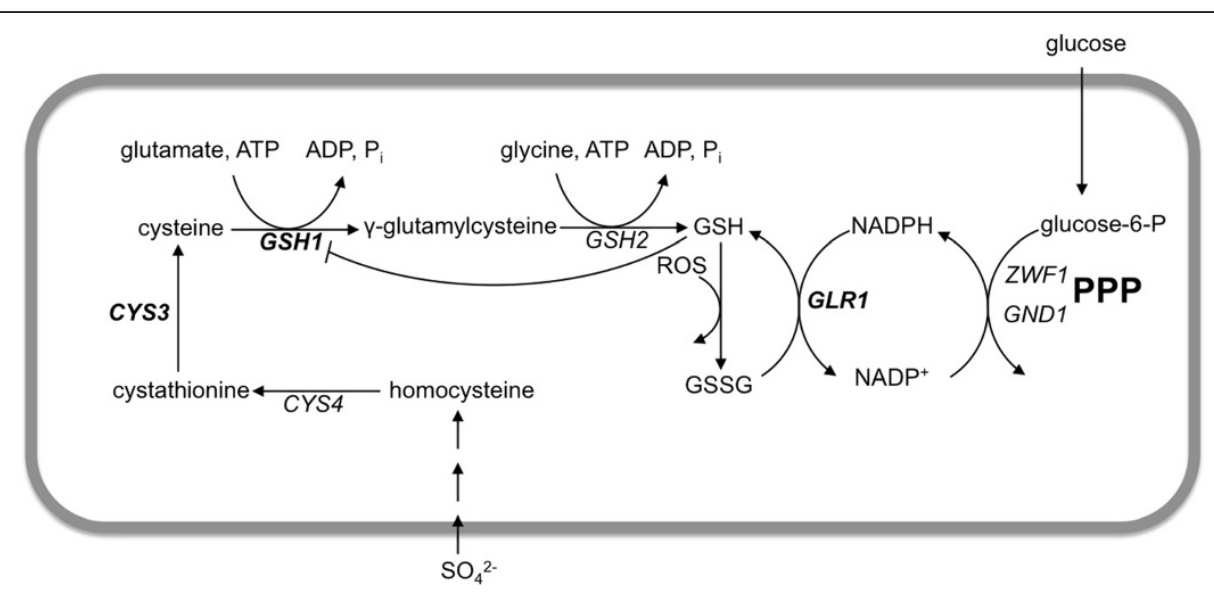

Figure 1 Simplified illustration on glutathione metabolism in S. cerevisiae. The genes overexpressed in the present study are marked in bold. For simplicity, only the major route for cytosolic NADPH production - PPP - is shown. 


\section{Results}

Several inhibitors generated during pretreatment of lignocellulosic biomass are known to influence the redox balance of the fermenting organism [24]. In the present study, the redox state of $S$. cerevisiae was modified by modulating the intracellular levels of glutathione by overexpressing three genes involved in glutathione biosynthesis and metabolism: GSH1, CYS3 and GLR1 (Figure 1). The mutant strains were then evaluated in simultaneous saccharification and fermentation of spruce.

\section{Growth performance and intracellular glutathione concentration of recombinant strains}

The strains constructed in the present study are shown in Table 1. The genes were put under the control of strong constitutive promoters of either the TDH3 or TPI1 genes and integrated into the genome of CEN.PK background strains. The maximum specific growth rate in mineral medium containing glucose as sole carbon source of the resulting strains is showed in Table 2 . In these conditions, no dramatic differences in specific growth rate were observed among the strains. CEN.PK 113-7D (wild type) showed the highest specific growth rate of $0.40 \pm 0.01 \mathrm{~h}^{-1}$, whereas the strain overexpressing GSH1/GLR1 showed the lowest specific growth rate of $0.34 \pm 0.00 \mathrm{~h}^{-1}$. All strains overexpressing GSH1 had a lower specific growth rate than the wild type whereas GLR1 overexpression alone did not influence growth kinetics in defined mineral medium.

To assess the effect of overexpression of GSH1, CYS3 and GLR1 on intracellular glutathione levels, total and oxidized glutathione were quantified in early exponential phase in cells grown in defined mineral medium (Figure 2). The concentration of GSH could then be deduced from the measured amounts. The strains overexpressing GSH1 and GSH1/CYS3 showed the highest increase in total glutathione concentration, accumulating $42 \%$ higher levels than the wild type. The oxidized glutathione levels (GSSG) were also higher for these recombinant strains. When GSH1/CYS3/GLR1 were overexpressed, a 39\% increase in total glutathione was observed, whereas overexpression of GSH1/GLR1 resulted in a $28 \%$ increase in the total glutathione levels. Overexpression of GLR1 alone did not alter the levels of total GSH compared to the wild type. The results are summarized in Table 2.

The intracellular levels of glutathione can be used to characterize the state of the intracellular redox environment. Two principal approaches have been used for this purpose: the [GSH]:[GSSG] ratio and the GSSG/2GSH half-cell potential $\left(\mathrm{E}_{\mathrm{hc}}\right)$.

The [GSH]:[GSSG] ratio varied considerably among the strains (Table 2). The highest ratio was observed in the wild type, where it was estimated to be 88 . Overexpression of GSH1 resulted in a decrease of the ratio to 37. Although glutathione reductase catalyzes the conversion of GSSG to GSH, overexpression of this gene did not result in a higher [GSH]:[GSSG] ratio than the wild type. In fact, the ratio was estimated to 67 , thus $24 \%$ lower than the value for CEN.PK 113-7D. Surprisingly, GSH1 overexpression in combination with GLR1 did not change the ratio compared to the strain where only GSH1 was overexpressed, and consequently it remained at a low level compared to the wild type strain. The lowest ratio (i.e. 32) was observed in the strain overexpressing GSH1 and CYS3. When all three genes were overexpressed in the same strain, the [GSH]:[GSSH] ratio was restored to a similar level as the strain overexpressing only GLR1, but the ratio was still significantly lower than for the wild type.

$E_{h c}$ can be calculated from the Nernst equation after determination of the intracellular concentrations of reduced and oxidized glutathione (see methods section). The estimated $E_{h c}$ values are listed in Table 2. The halfcell redox potentials for the strains overexpressing GSH1/GLR1 and GSH1/CYS3 were significantly higher than the wild type, indicating a more oxidizing environment in these strains. The $E_{h c}$ values estimated for the other strains were not statistically different from the wild type.

\section{Evaluation of strains in simultaneous saccharification and fermentation}

Simultaneous saccharification and fermentation (SSF) has been proposed as an attractive process concept for

Table 1 Strains used and constructed in the study

\begin{tabular}{|c|c|c|c|}
\hline $\begin{array}{l}\text { Strain } \\
\text { background }\end{array}$ & $\begin{array}{l}\text { Recombinant } \\
\text { strain }\end{array}$ & Genotype & Reference \\
\hline CEN.PK 113-7D & - & MATa, MAL2-8C, SUC2 & {$[25]$} \\
\hline CEN.PK 113-5D & GSH1 & ura3-52::URA3-TDH3p-GSH1-CYC1t, MATa, MAL2-8c, SUC2 & This study \\
\hline CEN.PK 102-3A & GSH1/CYS3 & ura3-52::URA3-TDH3p-GSH1-CYC1t, leu2-3,112::LEU2-TPI1-CYS3-CYC1t, MATa, MAL2-8c, SUC2 & This study \\
\hline CEN.PK 113-3C & GLR1 & trp 1-289::TRP1-TDH3p-GLR1-CYC1t, MATa, MAL2-8c, SUC2 & This study \\
\hline CEN.PK 113-9D & GSH1/GLR1 & ura3-52::URA3-TDH3p-GSH1-CYC1t, trp1-289::TRP1-TDH3p-GLR1-CYC1t, MATa, MAL2-8', SUC2 & This study \\
\hline CEN.PK 113-6B & GSH1/CYS3/GLR1 & $\begin{array}{l}\text { ura3-52::URA3-TDH3p-GSH1-CYC1t, leu2-3,112::LEU2-TPI1-CYS3-CYC1t, trp1-289::TRP1-TDH3p-GLR1-CYC1t } \\
\text { MATa, MAL2-8', SUC2 }\end{array}$ & This study \\
\hline
\end{tabular}


Table 2 Maximum specific growth rate and intracellular concentrations of total, reduced and oxidized glutathione in the strains in this study

\begin{tabular}{|c|c|c|c|c|c|c|}
\hline Strain & $\mu_{\max }\left(h^{-1}\right)$ & Total glutathione $\left(\mu \mathrm{mol} \mathrm{g} \mathrm{DW}{ }^{-1}\right)$ & GSH $\left(\mu \mathrm{mol} \mathrm{g} \mathrm{DW} W^{-1}\right)$ & GSSG $\left(\mu \mathrm{mol} \mathrm{g} \mathrm{DW}{ }^{-1}\right)$ & $\mathrm{E}_{\mathrm{hc}}(\mathrm{mV})$ & [GSH]:[GSSG] \\
\hline CEN.PK 113-7D & $0.40 \pm 0.01$ & $8.8 \pm 0.7$ & $8.7 \pm 0.7$ & $0.10 \pm 0.01$ & $-225 \pm 2$ & $88 \pm 10$ \\
\hline GSH1 & $0.36 \pm 0.01$ & $12.5 \pm 2.0$ & $12.2 \pm 2.0$ & $0.33 \pm 0.03$ & $-218 \pm 5$ & $37 \pm 8$ \\
\hline GSH1/CYS3 & $0.37 \pm 0.00$ & $12.5 \pm 0.3$ & $12.1 \pm 0.3$ & $0.38 \pm 0.04$ & $-216 \pm 1$ & $32 \pm 3$ \\
\hline GLR1 & $0.39 \pm 0.00$ & $8.9 \pm 1.1$ & $8.8 \pm 1.1$ & $0.13 \pm 0.01$ & $-221 \pm 4$ & $67 \pm 9$ \\
\hline GSH1/GLR1 & $0.34 \pm 0.00$ & $11.3 \pm 1.4$ & $11.0 \pm 1.4$ & $0.29 \pm 0.03$ & $-217 \pm 2$ & $37 \pm 1$ \\
\hline GSH1/CYS3/GLR1 & $0.37 \pm 0.01$ & $12.2 \pm 0.3$ & $12.0 \pm 0.2$ & $0.19 \pm 0.03$ & $-225 \pm 1$ & $62 \pm 7$ \\
\hline
\end{tabular}

The values given are mean values of triplicate cultivations \pm standard deviation.

lignocellulosic bioethanol production [26,27]. In this process, the enzymatic hydrolysis occurs concomitantly with the fermentation in the same reaction vessel. The advantages result from the low end-product inhibition of cellulolytic enzymes, the short process time and the lower investment costs compared to separate hydrolysis and fermentation (SHF) [27]. The recombinant strains constructed in the present study were evaluated in SSF of pretreated spruce, to investigate if the engineering strategy resulted in increased robustness at process-like conditions. Spruce was chosen as it has been shown to be one of the most challenging materials in terms of inhibitor content $[28,29]$. As the cellulolytic enzymes generally require higher temperatures than the microbes, the process was carried out at $35^{\circ} \mathrm{C}$. The composition of the pretreated raw material is shown in Table 3 and a time course of glucose and ethanol concentrations during the SSF are shown in Figure 3. All strains started to consume glucose and produce ethanol instantly after inoculation. All recombinant strains with increased intracellular glutathione levels, that is GSH1, GSH1/CYS3, GSH1/GLR1 and GSH1/ CYS3/GLR1, sustained ethanol production for 10 hours, with consistent decrease in free glucose concentration (Figure 3). In contrast, glucose started to accumulate in the cultures with the strain overexpressing GLR1 and the wild type after 4 and 6 hours respectively, as a result of the sugar release rate from the lignocellulosic material superseding the glucose consumption rate of the microorganism. As a consequence, the highest ethanol concentration was achieved in the cultivation with the strain overexpressing both GSH1 and GLR1, which reached $14.0 \mathrm{~g} \mathrm{~L}^{-1}$ ethanol after 48 hours. The strains overexpressing GSH1, GSH1/CYS3 and GSH1/CYS3/ GLR1 produced 13.7, 13.1 and $12.3 \mathrm{~g} \mathrm{~L}^{-1}$ ethanol, respectively, after 48 hours. The lowest maximal ethanol concentration was achieved in the cultivation with the strain overexpressing GLR1 (6.0 $\mathrm{g} \mathrm{L}^{-1}$ at 48 hours), followed by the wild type that reached $8.2 \mathrm{~g} \mathrm{~L}^{-1}$ at 48 hours. The maximum ethanol concentrations achieved and the ethanol yields on hexoses are reported in Table 4.

In order to study how the specific production rate of ethanol varied during the course of the fermentation, this parameter was calculated between each sampling point (Figure 4). The specific production rate of ethanol

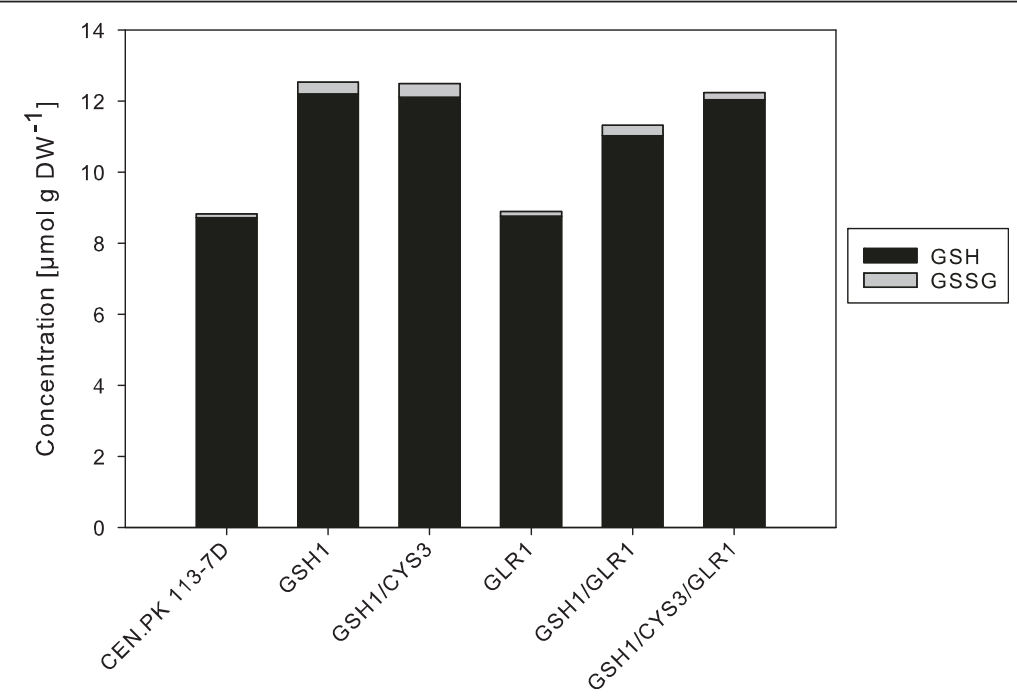

Figure 2 Intracellular levels of reduced (GSH) and oxidized (GSSG) glutathione. The bars represent mean values from triplicate cultivations. 
Table 3 Composition of pretreated spruce at $10 \%(w / w)$ WIS

\begin{tabular}{|c|c|}
\hline Component & Concentration $\left[\mathrm{g} \mathrm{L}^{-1}\right]$ \\
\hline \multicolumn{2}{|l|}{ Solid fraction } \\
\hline Glucose & 51.9 \\
\hline Mannose & 0.3 \\
\hline Xylose & 0.2 \\
\hline \multicolumn{2}{|l|}{ Liquid fraction } \\
\hline Glucose & 17.6 \\
\hline Mannose & 11.6 \\
\hline Xylose & 5.2 \\
\hline Galactose & 2.4 \\
\hline Arabinose & 1.6 \\
\hline Acetic acid ${ }^{a}$ & 3.3 \\
\hline Formic acid ${ }^{a}$ & 0.1 \\
\hline Levulinic acid $^{a}$ & 0.8 \\
\hline $\mathrm{HMF}^{\mathrm{a}}$ & 2.3 \\
\hline Furfural $^{a}$ & 1.4 \\
\hline
\end{tabular}

decreased during the course of the process, and was equal to 0 after 48 hours of fermentation for all strains. The highest initial production rate was observed in the cultivation with the strain overexpressing GSH1 between 0 and 2 hours. In general, all strains harboring the GSH1 overexpression construct maintained a higher specific production rate of ethanol than the wild type and the
GLR1 overexpressing strain during the first 48 hours of cultivation.

Both HMF and furfural were converted to different extent by the investigated strains in the SSF cultivation performed in the present study (Figure 5). The conversion of both furan aldehydes were active as long as the cells were viable and consumed sugars. All four strains overexpressing GSH1 converted the largest amount of HMF and furfural during the course of the cultivation, thus outperforming the wild type strain. Similar results were also obtained in cultivations using a synthetic hydrolysate (data not shown). The strain overexpressing GLR1 converted the lowest amount of HMF and furfural in the present study.

\section{Discussion}

In the present work we show that by increasing the intracellular concentration of glutathione, strain robustness could be improved in industrially relevant conditions posed by pretreated spruce in an SSF process. As a mechanism for the improved robustness, we hypothesize a redox buffering capacity potentiating effect, originating from the increased availability of reduced glutathione. The enhanced redox buffering capacity would confer higher inherent capability of the intracellular environment to titrate cofactor consuming exogenous molecules. Indeed, at the time of writing this article, it was reported that furan aldehydes, and in particular furfural, depleted the intracellular pool of glutathione. This fact further corroborates the hypothesis of the redox potentiating effects of increasing

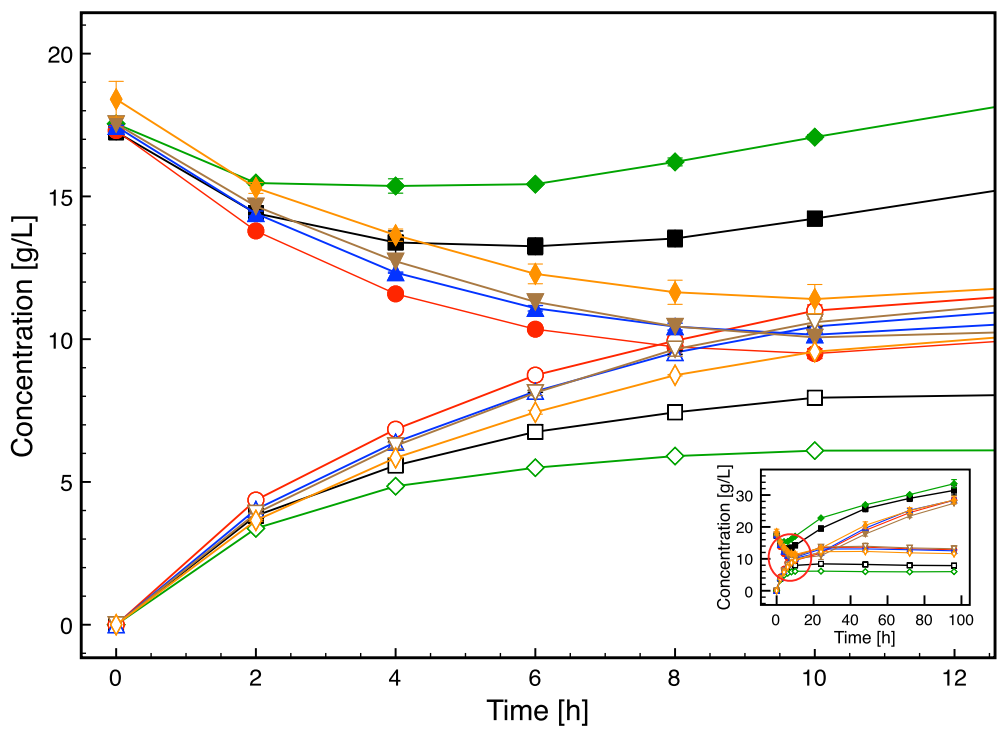

Figure 3 Time course of glucose and ethanol concentrations in SSF of spruce with the strains investigated in the present study. Black squares: CEN.PK 113-7D, red circles: GSH1, blue triangles: GSH1/CYS3, green diamonds: GLR1, brown reversed triangles: GSH1/GLR1, orange stretched diamonds: GSH1/CYS3/GLR1. The filled and open symbols represent glucose and ethanol, respectively. The data points are mean values from two independent cultivations. The error bars represent the maximum and minimum values. 
Table 4 Results from SSF of pretreated spruce with the strains constructed in the present study

\begin{tabular}{lll}
\hline Strain & $\left.\begin{array}{l}\text { Ethanol yield on } \\
\text { hexoses }\end{array} \mathbf{~} \mathbf{g ~ g}^{-1}\right)$ & $\begin{array}{l}\text { Ethanol concentration } \\
\text { at } \mathbf{4 8} \mathbf{~} \mathbf{( \mathbf { g ~ L } ^ { - 1 } )}\end{array}$ \\
\hline CEN.PK 113-7D & $0.10 \pm 0.00$ & $8.2 \pm 0.0$ \\
GSH1 & $0.16 \pm 0.00$ & $13.7 \pm 0.2$ \\
GSH1/CYS3 & $0.16 \pm 0.00$ & $13.1 \pm 0.3$ \\
GLR1 & $0.07 \pm 0.00$ & $6.0 \pm 0.0$ \\
GSH1/GLR1 & $0.17 \pm 0.01$ & $14.0 \pm 1.0$ \\
GSH1/CYS3/GLR1 & $0.14 \pm 0.00$ & $12.3 \pm 0.2$
\end{tabular}

${ }^{a}$ The ethanol yield was calculated based on the total amount of hexoses present in the solid and liquid fraction of the pretreated raw material at the start of the SSF.

The values given are mean values from duplicate cultivations \pm standard deviation

the intracellular concentration of GSH [11]. In the same paper, it was shown that overexpression of GSH1 resulted in a decreased lag-phase of growth in the presence of furfural, whereas no effect was observed in the case of HMF. In fact, similar strategies, such as overproduction of the intracellular protective metabolite and ROS scavenger ascorbic acid, have been shown to increase robustness to various environmental stresses [30]. It is known that inhibitors such as HMF and furfural not only drain microorganisms of reducing power, but have also been shown to induce the generation of reactive oxygen species in $S$. cerevisiae [5,10].

Quantification of the [GSH]:[GSSG] ratio and the half-cell redox potential $\mathrm{E}_{\mathrm{hc}}$ were used to assess the magnitude of the changes in intracellular redox environment upon overexpressing genes involved in the glutathione biosynthesis pathway. $E_{\mathrm{hc}}$, which is calculated from the Nernst equation (see Methods section), has been reported to be a more reliable method, as the stoichiometry of the glutathione redox couple (GSSG $+2 \mathrm{H}^{+}+2 \mathrm{e}^{-} \rightarrow 2 \mathrm{GSH}$ ) is taken into account $[13,15,31]$. $\mathrm{E}_{\mathrm{hc}}$ is thus dependent on both the [GSH]:[GSSG] ratio and the absolute concentration of GSH, which means that cells having the same [GSH]: [GSSG] ratios can have different redox potentials depending on the concentration of GSH. Consequently, cells with a high concentration of intracellular reduced glutathione have a higher buffering capacity against oxidative insults than cells with a lower concentration of reduced glutathione.

Although overexpression of GSH1 increased the intracellular levels of reduced glutathione, unexpectedly none of the recombinant strains had a more reducing intracellular environment compared to the wild type when grown in defined mineral medium (as it would be indicated by a higher [GSH]:[GSSG] ratio and/or a lower $\mathrm{E}_{\mathrm{hc}}$ ). In fact, the ultimate reason for the higher estimates of $E_{h c}$ in these strains was indeed the higher intracellular concentration of GSSG in all recombinant strains harboring the GSH1 overexpression construct. However, it has been shown in a recent study [32] that the cytosolic glutathione redox potential was independent of changes in whole-cell GSSG levels due to compartmentalization of GSSG. The GSSG concentration was maintained at very low levels in the cytosol through the action of Ycflp pumping GSSG to the vacuole. Hence, calculating the $\mathrm{E}_{\mathrm{hc}}$ using GSSG levels determined from whole cell-extracts as in the present study in fact overestimates the cytosolic redox potential. Moreover, it has recently been demonstrated that significant differences exist in the redox potential between different cellular compartments [33]. At a purely speculative level, assuming an oxidation level of $0.03 \%$ of the cytosolic glutathione pool (as estimated by Østergaard et al. [34]) for all strains, the estimated $E_{h c}$ values for all recombinant strains overexpressing GSH1 would in fact be lower than those in

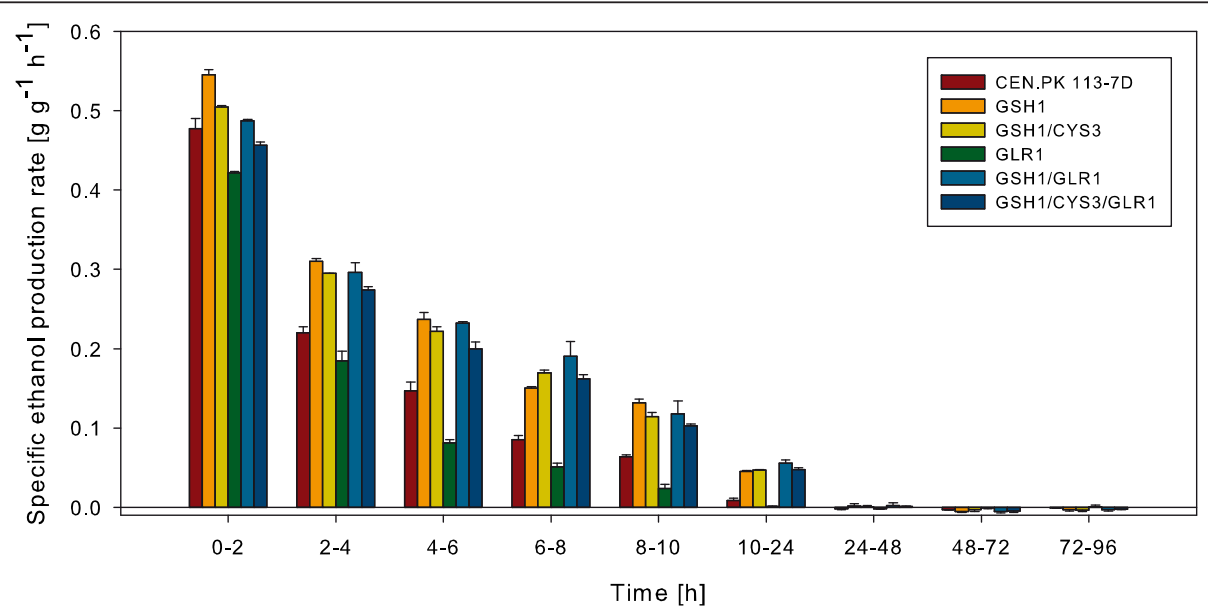

Figure 4 Specific ethanol production rates over time in SSF of spruce with the strains investigated in the present study. The specific rates were calculated assuming a cell concentration of $4 \mathrm{~g} \mathrm{~L}^{-1}$ throughout the whole fermentation course. The bars represent mean values from two independent cultivations. The error bars show the maximum and minimum values. 


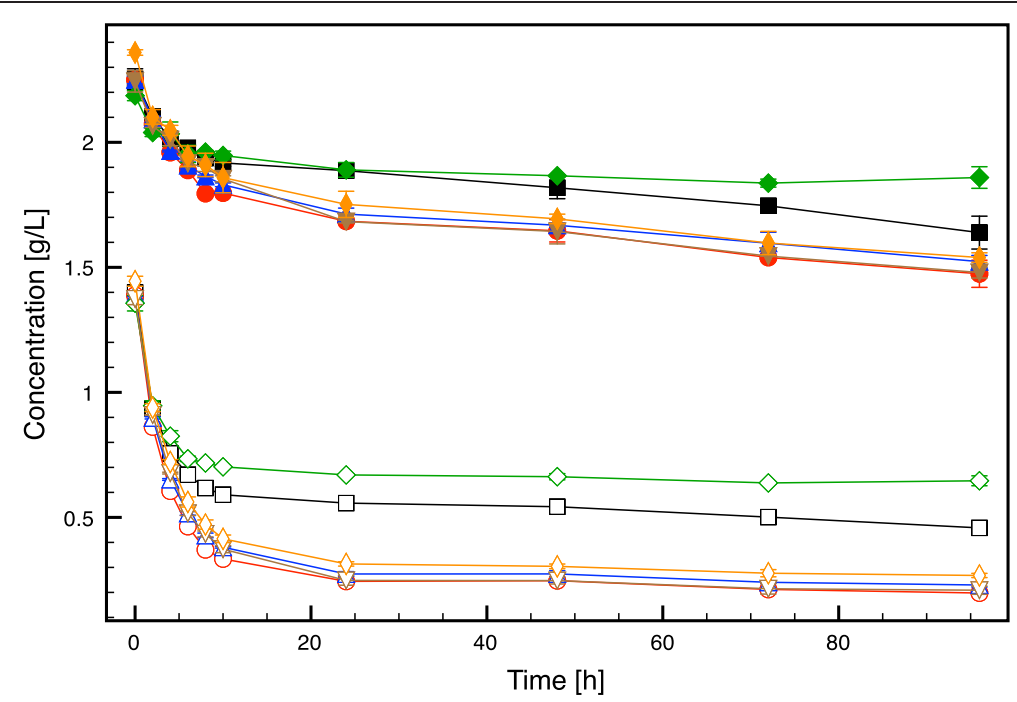

Figure 5 Time course of HMF and furfural concentrations in SSF of spruce with the strains investigated in the present study. Black squares: CEN.PK 113-7D, red circles: GSH1, blue triangles: GSH1/CYS3, green diamonds: GLR1, brown reversed triangles: GSH1/GLR1, orange stretched diamonds: GSH1/CYS3/GLR1. The filled and open symbols represent HMF and furfural, respectively. The data points are mean values from two independent cultivations. The error bars represent the maximum and minimum values.

the wild type ( $-277 \mathrm{mV}$ versus $-273 \mathrm{mV}$ ), which then would indicate that GSH1 overexpression increases the redox buffering capacity and consequently strain robustness.

The increased redox buffering capacity of the strains overexpressing GSH1 was evident in SSF of pretreated spruce at $10 \%(\mathrm{w} / \mathrm{w})$ water insoluble solids (WIS) concentration, which contains a spectrum of inhibitory compounds and in particular significant amounts of HMF and furfural (Table 3) [24]. All strains carrying the GSH1 overexpression construct were able to consume glucose and convert HMF and furfural for a longer period of time resulting in higher ethanol yields for these strains than the wild type. As overexpression of CYS3 in combination with GSH1 did not result in higher intracellular concentrations of glutathione than overexpression of GSH1 alone, the performance of this strain did not differ form the latter. An explanation could be that the high GSH levels obtained by GSH1 overexpression prevent a further increase of the intracellular glutathione concentration, since it is known that Gsh1p is feedback inhibited by GSH [35]. Unexpectedly, overexpression of GLR1 in combination with GSH1 did not result in decreased levels of GSSG, possibly due to limitation of NADPH. Consequently, the GSH1/GLR1 strain performance was equivalent to that of the strain overexpressing GSH1. In contrast, GLR1 overexpression alone was found to be a burden for the cells when cultivated in the spruce slurry, which was manifested in an earlier cessation of ethanol production than the wild type. Competition for NADPH between Glr1p and furan aldehyde detoxifying oxidoreductases could be a possible explanation for this observation.
Although integration of the overexpression constructs influenced the maximum specific growth rate, the difference between the strains was not severe. All recombinant strains carrying the GSH1 overexpression construct exhibited a lower specific growth rate than the wild type, and the strain overexpressing both GSH1 and GLR1 grew at the lowest specific growth rate. The lower specific growth rates can be explained by a combination of metabolic burden on the cell imposed by the overexpression of the introduced genetic constructs, and uncharacterized effects on strain growth behavior commonly encountered as a consequence of using auxotrophic markers. There is also a possibility that the decrease in specific growth rate in the strains overexpressing GSH1 is connected to partial depletion of cysteine due to overproduction of glutathione. In fact, overexpression of CYS3 somewhat restored the specific growth rate in strains overexpressing GSH1 (Table 2). On the other hand, these small changes in specific growth rate may be of minor importance for industrial bioethanol production, since the microorganisms are thought to reside in a non-growing state in a process such as SSF [36]. Therefore, an engineering strategy that results in a small decrease in specific growth rate may not be unfavorable as long as the microorganism is more tolerant to the process conditions.

Overall, the results from this study show that engineering of S. cerevisiae with increased intracellular glutathione production has a beneficial effect on strain robustness by extending the time of survival in an SSF process using a challenging substrate. The mechanism for the increased 
tolerance supposedly originates from an increased redox buffering capacity resulting from the increased pool of reduced glutathione in the recombinant strains.

\section{Conclusions}

In this work, we showed that engineering of the redox metabolism of $S$. cerevisiae in terms of increasing the intracellular levels of glutathione by overexpressing GSH1 resulted in increased strain robustness in an SSF process. This was reflected in higher cell survival and final ethanol concentrations of the recombinant strains compared to the wild type in industrial media.

\section{Methods}

\section{Strains}

The strains used in the present study are listed in Table 1. CEN.PK 113-7D (MATa, MAL2-8 ${ }^{c}$, SUC2) was used as reference strain.

\section{Plasmid construction}

GSH1 was amplified from genomic DNA of CEN.PK 113-7D using High-Fidelity DNA Polymerase (Thermo Fisher Scientific) with primers GSH1_SalI_fw (5' ATAAGTCGACATGGGACTCTTAGCTTTGGG) and GSH1_KpnI_rev (5' GCATGGTACCTTAACATTTGCT TTCTATTGAAG) and cloned into the integrative plasmid YIplac211 [37] harboring the promoter TDH3p, the terminator CYC1t and a functional copy of URA3. CYS3 was codon-optimized with JCat [38] and ordered subcloned into YIplac128 (containing a functional copy of LEU2) with TPI1p and CYC1t from Genscript (Piscataway, NJ, USA). GLR1 was amplified from genomic DNA of CEN.PK 113-7D with High-Fidelity DNA Polymerase (Thermo Fisher Scientific) with primers GLR1_XbaI_fw (5'TCTAGAATGCTTTCTGCAACCAAACAAAC) and GLR1_SmaI_rev (5' ACCCGGGTCATCTCATAGTAA CCAAT TCT TC) and cloned into the integrative plasmid YIplac204 harboring TDH3p, CYC1t and a functional copy of TRP1. The constructs were verified by DNA sequencing.

\section{Strain construction}

All yeast transformations were performed according to [39]. CEN.PK 113-5D, CEN.PK 102-3A, CEN.PK 113-9D and CEN.PK 113-6B were transformed with YIplac211TDH3p-GSH1-CYC1t previously linearized in URA3 with StuI. Selection was done on Yeast Nitrogen Base (YNB) plates with addition of relevant amino acids when applicable. CEN.PK 102-3A and CEN.PK 113-6B containing the overexpression construct for GSH1 were subsequently transformed with YIplac128-TPIP-CYS3-CYC1t previously linearized in LEU2 with ClaI and selected on YNB agar plates with supplementation of amino acids when applicable. CEN.PK 113-3C, CEN.PK 113-9D (containing GSH1 overexpression construct) and CEN.PK 113-6B (containing GSH1 and CYS3 overexpression constructs) were transformed with YIplac204-TDH3p-GLR1-CYC1t previously linearized in TRP1 with BstXI and selected on YNB agar plates with supplementation of amino acids when applicable. All integrations were verified by PCR.

\section{Specific growth rate determination and quantification of intracellular glutathione}

Single colonies of the respective strains were picked from YPD plates and inoculated in $5 \mathrm{~mL}$ defined mineral medium according to [40] containing $20 \mathrm{~g} \mathrm{~L}^{-1}$ glucose and $50 \mathrm{mM}$ potassium hydrogen phthalate. After 24 hours, $50 \mathrm{~mL}$ medium of the same composition was inoculated at a final $\mathrm{OD}_{600}$ of 0.2. Samples were taken at regular intervals during the exponential phase and the maximum specific growth rate was determined from the slope of $\ln$ $\left(\mathrm{OD}_{600}\right)$ plotted against time (h). The final $\mathrm{OD}_{600}$ at the end of the exponential phase was approximately 5 . In early exponential phase (when $\mathrm{OD}_{600}$ was 1.1-1.5), $5 \mathrm{~mL}$ samples were withdrawn and centrifuged at $2400 \mathrm{~g}$ for $4 \mathrm{mi}-$ nutes at $4^{\circ} \mathrm{C}$. Total glutathione was determined according to $[32,41]$. The cells were washed with $0.1 \mathrm{M}$ potassium phosphate buffer, $\mathrm{pH} 7.5$, resuspended in ice-cold $8 \mathrm{mM}$ $\mathrm{HCl}, 1.3 \%(\mathrm{w} / \mathrm{v}) 5$-sulfosalicylic acid solution and disintegrated with glass beads (particle size $425-600 \mu \mathrm{m}$ ) in a bead mill for $3 * 30 \mathrm{~s}$ at maximum speed. The lysate was centrifuged at $10000 \mathrm{~g}$ for 5 minutes at $4{ }^{\circ} \mathrm{C}$ and the supernatant was used for total glutathione estimation. Samples for oxidized glutathione quantification were prepared by letting $100 \mu \mathrm{L}$ extract of the preparation above react with $2 \mu \mathrm{L} \mathrm{20 \%} \mathrm{(v/v)} \mathrm{2-vinylpyridine} \mathrm{in} \mathrm{ethanol.} \mathrm{The}$ $\mathrm{pH}$ was brought to 7 by addition of $40 \mu \mathrm{L} 1 \mathrm{M}$ Mes/Tris buffer at $\mathrm{pH} 7$ and the reaction took place at room temperature for 1 hour. Final concentrations in the reaction mix were the following: $1.2 \mathrm{IU} / \mathrm{mL}$ GSH reductase; 0.73 mM DTNB; $0.24 \mathrm{mM}$ NADPH; 0.09\% 5-sulfosalicylic acid. Standard curves were constructed using reduced glutathione or oxidized glutathione, respectively, ranging from 0.206 to $26.4 \mu \mathrm{M}$. The assay was performed in 96well plates in a Fluostar microplate reader (BMG Labtech $\mathrm{GmbH}$, Offenburgh, Germany) reading absorbance at $412 \mathrm{~nm}$ after automatic addition of NADPH to start the reaction. The absorbance was read every $5 \mathrm{~s}$ for $2 \mathrm{~min}$ in each reaction.

\section{Calculation of intracellular redox environment}

The intracellular redox environment was estimated from the Nernst equation according to [13] using the intracellular concentrations of GSH and GSSG. An intracellular volume of $2.38 \mathrm{~mL} \mathrm{gDW}^{-1}$ was assumed when calculating the intracellular concentrations [42] (visual inspection under microscope did not reveal any significant differences in cell size between the strains). 


$$
E_{h c}=E_{0}-2.303 \frac{R T}{n F} \log _{10} \frac{[G S H]^{2}}{[G S S G]}
$$

where $E_{0}$ is the standard potential for GSH $(-240 \mathrm{mV})$ at $\mathrm{pH} 7$ [43], $\mathrm{R}$ is the gas constant $\left(8.31 \mathrm{~J} \mathrm{~mol}^{-1} \mathrm{~K}^{-1}\right)$, $\mathrm{T}$ is the absolute temperature $(303 \mathrm{~K}), \mathrm{n}$ is the number of electrons exchanged in the process (2) and $\mathrm{F}$ is the Faraday constant $\left(96485 \mathrm{C} \mathrm{mol}^{-1}\right)$.

\section{Simultaneous saccharification and fermentation (SSF)}

Precultures were prepared by inoculating a single colony of the respective strain in $5 \mathrm{~mL}$ defined mineral medium according to [40] containing $50 \mathrm{~g} \mathrm{~L}^{-1}$ glucose and twice the amounts of the other components. After 24 hours, the first preculture was harvested and inoculated at a final OD of 0.2 in the above mentioned medium. The second preculture was harvested after all glucose had been consumed, which was monitored with glucose test strips (Keto-Diabur-Test 5000, Roche, Basel, Switzerland). SSF was carried out at $50 \mathrm{~g}$ working weight in shake-flasks with steam-pretreated spruce provided by SEKAB E-Technology (Örnsköldsvik, Sweden) at $10 \%(\mathrm{w} / \mathrm{w})$ water insoluble solids (WIS) content. The slurry was supplemented with $1 \mathrm{~g} \mathrm{~L}^{-1}$ yeast extract, $0.5 \mathrm{~g} \mathrm{~L}^{-1}\left(\mathrm{NH}_{4}\right)_{2} \mathrm{HPO}_{4}, 0.025 \mathrm{~g} \mathrm{~L}^{-1} \mathrm{MgSO}_{4} 7 \mathrm{H}_{2} \mathrm{O}$ and $50 \mathrm{mM}$ potassium phthalate. The SSF experiment was initiated by addition of cell suspension yielding a final cell concentration of $4 \mathrm{~g} \mathrm{~L}^{-1} \mathrm{DW}$ and by addition of Celluclast 1.5 L (Novozymes A/S, Bagsvaerd, Denmark) and Novozyme 188 (Novozymes A/S, Bagsvaerd, Denmark) at $10 \mathrm{mg}$ protein/g WIS and $500 \mathrm{nkat} / \mathrm{g}$ WIS, respectively. Samples were withdrawn regularly throughout the cultivations, which were performed in duplicates at $35^{\circ} \mathrm{C}$ and $\mathrm{pH} 5$.

\section{Quantification of sugars and extracellular metabolites}

Samples from SSF experiments were centrifuged at $14000 \mathrm{~g}$ for 2 minutes and filtered through $0.2 \mu \mathrm{m}$ nylon membranes. The samples were stored at $-20^{\circ} \mathrm{C}$ until analysis. Concentrations of glucose, xylose, mannose, galactose, ethanol, glycerol, xylitol, HMF and furfural were analyzed using an HPLC system (Dionex, Sunnyvale, CA) equipped with an Aminex HPX87-P column (Bio-Rad Laboratories, Munich, Germany) operated at $85^{\circ} \mathrm{C}$ with milliQ- $\mathrm{H}_{2} \mathrm{O}$ as eluent at a flow rate of $0.6 \mathrm{~mL} \mathrm{~min}{ }^{-1}$. Detection of sugars and alcohols were made with a Shodex RI-101 refractive index detector (Showa Denko, New York, NY). HMF and furfural were detected with an UV detector at $210 \mathrm{~nm}$ (Dionex, Sunnyvale, CA).

\section{Determination of cell mass}

Cell mass was determined according to [44]. $5 \mathrm{~mL}$ of culture broth was filtered through a $0.45 \mu \mathrm{m}$ PES membrane (Sartorius Stedim, Aubagne, France). The filters were washed with MilliQ- $\mathrm{H}_{2} \mathrm{O}$ and dried in a microwave oven at $120 \mathrm{~W}$ for 15 minutes and subsequently weighed.

\section{Competing interests}

The authors declare that they have no competing interests.

\section{Authors' contributions}

MA participated in the design of the study, constructed the strains, performed the experiments and wrote the manuscript. VM participated in the design of the study, participated in the strain construction and commented on the manuscript. $\mathrm{HH}$ participated in the strain construction. LO participated in the design of the study and commented on the manuscript. MB participated in the design of the study and commented on the manuscript. All authors read and approved the final manuscript.

\section{Acknowledgements}

This study was performed in NEMO, a project under EU's $7^{\text {th }}$ framework program, grant number 222699. SEKAB E-Technology is gratefully acknowledged for providing the pretreated spruce raw material.

\section{Author details}

${ }^{1}$ Department of Chemical and Biological Engineering, Industrial Biotechnology, Chalmers University of Technology, Gothenburg SE-41296, Sweden. ${ }^{2}$ Current affiliation: Lehrstuhl für Biotechnologie, RWTH Aachen University, Aachen D-52074, Germany.

Received: 10 July 2013 Accepted: 26 September 2013 Published: 1 October 2013

\section{References}

1. Attfield PV: Stress tolerance: the key to effective strains of industrial baker's yeast. Nat Biotechnol 1997, 15:1351-1357.

2. Klinke $H$, Thomsen A, Ahring B: Inhibition of ethanol-producing yeast and bacteria by degradation products produced during pre-treatment of biomass. Appl Microbiol Biotechnol 2004, 66:10-26.

3. Almeida JRM, Bertilsson M, Gorwa-Grauslund MF, Gorsich S, Liden G: Metabolic effects of furaldehydes and impacts on biotechnological processes. Appl Microbiol Biotechnol 2009, 82:625-638.

4. Modig T, Liden G, Taherzadeh MJ: Inhibition effects of furfural on alcohol dehydrogenase, aldehyde dehydrogenase and pyruvate dehydrogenase. Biochem J 2002, 363:769-776.

5. Allen SA, Clark W, McCaffery JM, Cai Z, Lanctot A, Slininger PJ, Liu ZL, Gorsich SW: Furfural induces reactive oxygen species accumulation and cellular damage in Saccharomyces cerevisiae. Biotechnol Biofuels 2010, 3:2.

6. Taherzadeh MJ, Gustafsson L, Niklasson C, Liden G: Conversion of furfural in aerobic and anaerobic batch fermentation of glucose by Saccharomyces cerevisiae. J Biosci Bioeng 1999, 87:169-174.

7. Palmqvist E, Almeida JS, Hahn-Hagerdal B: Influence of furfural on anaerobic glycolytic kinetics of Saccharomyces cerevisiae in batch culture. Biotechnol Bioeng 1999, 62:447-454.

8. Morimoto S, Murakami M: Studies on fermentation products from aldehyde by microorganisms: the fermentative production of furfural alcohol from furfural by yeasts (part I). J Ferment Technol 1967, 45:442-446.

9. Liu ZL, Slininger PJ, Dien BS, Berhow MA, Kurtzman CP, Gorsich SW: Adaptive response of yeasts to furfural and 5-hydroxymethylfurfural and new chemical evidence for HMF conversion to 2,5-bishydroxymethylfuran. J Ind Microbiol Biotechnol 2004, 31:345-352.

10. Ask M, Bettiga M, Mapelli $V$, Olsson L: The influence of HMF and furfural on redox-balance and energy-state of xylose-utilizing Saccharomyces cerevisiae. Biotechnol Biofuels 2013, 6:22.

11. Kim D, Hahn JS: Roles of the Yap1 Transcription Factor and Antioxidants in Saccharomyces cerevisiae's Tolerance to Furfural and 5Hydroxymethylfurfural, Which Function as Thiol-Reactive Electrophiles Generating Oxidative Stress. App/ Environ Microbiol 2013, 79:5069-5077.

12. Grant CM: Role of the glutathione/glutaredoxin and thioredoxin systems in yeast growth and response to stress conditions. Mol Microbiol 2001, 39:533-541.

13. Schafer FQ, Buettner GR: Redox environment of the cell as viewed through the redox state of the glutathione disulfide/glutathione couple. Free Radic Biol Med 2001, 30:1191-1212. 
14. Penninckx MJ: An overview on glutathione in Saccharomyces versus nonconventional yeasts. FEMS Yeast Res 2002, 2:295-305.

15. Drakulic T, Temple MD, Guido R, Jarolim S, Breitenbach M, Attfield PV, Dawes IW: Involvement of oxidative stress response genes in redox homeostasis, the level of reactive oxygen species, and ageing in Saccharomyces cerevisiae. FEMS Yeast Res 2005, 5:1215-1228.

16. Collinson LP, Dawes IW: Isolation, characterization and overexpression of the yeast gene, GLR1, encoding glutathione reductase. Gene 1995, 156:123-127.

17. Rietsch A, Beckwith J: The genetics of disulfide bond metabolism. Annu Rev Genet 1998, 32:163-184.

18. Kim IS, Kim YS, Yoon HS: Glutathione reductase from Oryza sativa increases acquired tolerance to abiotic stresses in a genetically modified Saccharomyces cerevisiae strain. J Microbiol Biotechnol 2012, 22:1557-1567.

19. Yoon HS, Shin SY, Kim YS, Kim IS: Glutathione reductase from Brassica rapa affects tolerance and the redox state but not fermentation ability in response to oxidative stress in genetically modified Saccharomyces cerevisiae. World J Microbiol Biotechnol 2012, 28:1901-1915.

20. Grant CM, Maclver FH, Dawes IW: Glutathione synthetase is dispensable for growth under both normal and oxidative stress conditions in the yeast Saccharomyces cerevisiae due to an accumulation of the dipeptide gamma-glutamylcysteine. Mol Biol Cell 1997, 8:1699-1707.

21. Collinson EJ, Wimmer-Kleikamp S, Gerega SK, Yang YH, Parish CR, Dawes IW, Stocker R: The yeast homolog of heme oxygenase-1 affords cellular antioxidant protection via the transcriptional regulation of known antioxidant genes. J Biol Chem 2011, 286:2205-2214

22. Alfafara C, Miura K, Shimizu H, Shioya S, Suga K: Cysteine addition strategy for maximum glutathione production in fed-batch culture of Saccharomyces cerevisiae. Appl Microbiol Biotechnol 1992, 37:141-146.

23. Nisamedtinov I, Kevvai K, Orumets K, Arike L, Sarand I, Korhola M, Paalme T: Metabolic changes underlying the higher accumulation of glutathione in Saccharomyces cerevisiae mutants. Appl Microbiol Biotechnol 2011, 89:1029-1037

24. Palmqvist $\mathrm{E}$, Hahn-Hägerdal B: Fermentation of lignocellulosic hydrolysates. II: inhibitors and mechanisms of inhibition. Bioresour Technol 2000, 74:25-33.

25. Entian KD, Kötter P: Yeast genetic strain and plasmid collections. In Methods in Microbiology. Edited by Stansfield I, Stark M. USA: Elsevier; 2007.

26. Gauss W, Suzuki S, Takagi M: Manufacture of alcohol from cellulosic materials using plural ferments. USA: Patent nr 3990944, Office USPaT; 1976.

27. Olofsson K, Bertilsson M, Lidén G: A short review on SSF - an interesting process option for ethanol production from lignocellulosic feedstocks. Biotechnol Biofuels 2008, 1:7.

28. Larsson S, Palmqvist E, Hahn-Hagerdal B, Tengborg C, Stenberg K, Zacchi G, Nilvebrant NO: The generation of fermentation inhibitors during dilute acid hydrolysis of softwood. Enzyme Microb Technol 1999, 24:151-159.

29. Larsson S, Reimann A, Nilvebrant NO, Jonsson LJ: Comparison of different methods for the detoxification of lignocellulose hydrolyzates of spruce. Appl Biochem Biotechnol 1999, 77-9:91-103.

30. Branduardi P, Fossati T, Sauer M, Pagani R, Mattanovich D, Porro D: Biosynthesis of vitamin $C$ by yeast leads to increased stress resistance. PLoS One 2007, 2:e1092.

31. Kirlin WG, Cai J, Thompson SA, Diaz D, Kavanagh TJ, Jones DP: Glutathione redox potential in response to differentiation and enzyme inducers. Free Radic Biol Med 1999, 27:1208-1218.

32. Morgan B, Ezerina D, Amoako TN, Riemer J, Seedorf M, Dick TP: Multiple glutathione disulfide removal pathways mediate cytosolic redox homeostasis. Nat Chem Biol 2013, 9:119-125.

33. Ayer A, Sanwald J, Pillay BA, Meyer AJ, Perrone GG, Dawes IW: Distinct redox regulation in sub-cellular compartments in response to various stress conditions in Saccharomyces cerevisiae. PLoS One 2013, 8:e65240.

34. Ostergaard H, Tachibana C, Winther JR: Monitoring disulfide bond formation in the eukaryotic cytosol. J Cell Biol 2004, 166:337-345.

35. Wheeler GL, Quinn KA, Perrone G, Dawes IW, Grant CM: Glutathione regulates the expression of gamma-glutamylcysteine synthetase via the Met4 transcription factor. Mol Microbiol 2002, 46:545-556.

36. Ask M, Olofsson K, Di Felice T, Ruohonen L, Penttilä M, Lidén G, Olsson L: Challenges in enzymatic hydrolysis and fermentation of pretreated Arundo donax revealed by a comparison between SHF and SSF. Process Biochem 2012, 47:1452-1459.
37. Gietz RD, Sugino A: New yeast-Escherichia coli shuttle vectors constructed with in vitro mutagenized yeast genes lacking six-base pair restriction sites. Gene 1988, 74:527-534.

38. Grote A, Hiller K, Scheer M, Munch R, Nortemann B, Hempel DC, Jahn D: JCat: a novel tool to adapt codon usage of a target gene to its potential expression host. Nucleic Acids Res 2005, 33:W526-W531.

39. Gietz RD, Woods RA: Yeast Transformation by the LiAc/SS Carrier DNA/ PEG Method. In Methods in Molecular Biology. Volume 313. Edited by Xiao W. Totowa, N.J: Humana Press Inc; 2005.

40. Verduyn C, Postma E, Scheffers W, Van Dijken J: Effect of benzoic acid on metabolic fluxes in yeasts: a continuous-culture study on the regulation of respiration and alcoholic fermentation. Yeast 1992, 8:501-517.

41. Rahman I, Kode A, Biswas SK: Assay for quantitative determination of glutathione and glutathione disulfide levels using enzymatic recycling method. Nat Protoc 2006, 1:3159-3165.

42. Ditzelmuller G, Wohrer W, Kubicek CP, Rohr M: Nucleotide pools of growing, synchronized and stressed cultures of Saccharomyces cerevisiae. Arch Microbiol 1983, 135:63-67.

43. Rost J, Rapoport S: Reduction-potential of glutathione. Nature 1964, 201:185

44. Olsson L, Nielsen J: Online and in situ monitoring of biomass in submerged cultivations. Trends Biotechnol 2009, 15:517-522.

doi:10.1186/1475-2859-12-87

Cite this article as: Ask et al:: Engineering glutathione biosynthesis of Saccharomyces cerevisiae increases robustness to inhibitors in pretreated lignocellulosic materials. Microbial Cell Factories 2013 12:87.

\section{Submit your next manuscript to BioMed Central and take full advantage of:}

- Convenient online submission

- Thorough peer review

- No space constraints or color figure charges

- Immediate publication on acceptance

- Inclusion in PubMed, CAS, Scopus and Google Scholar

- Research which is freely available for redistribution 